\title{
Research on the Incidence Rate of Extremely Preterm Birth in China and Relationship between Extremely Preterm Birth and Chorioamnionitis
}

\author{
LI YIN QIU, JIAN YING YAN*, HUI LIU AND KUN HAI REN \\ Department of Obstetrical, Fujian Provincial Maternity and Children's Hospital, affiliated Hospital of Fujian Medical University, \\ No.18 Daoshan Road, Fuzhou, Fujian 350001, China
}

Qiu et al.: Incidence Rate of Extremely Preterm Birth and its Relationship with Chorioamnionitis

\begin{abstract}
To investigate the relationship between the extremely preterm birth and chorioamnionitis based on Chinese population. Retrospective analysis was performed to evaluate 164 cases hospitalized in Fujian Provincial Maternity and Children's Hospital during $1^{\text {st }}$ January 2017 and $31^{\text {st }}$ June 2018. The clinical symptoms of the participators were recorded and the laboratory tests were performed. The incidence of the extremely preterm birth was $0.7 \%$ accounting for $7.2 \%$ in the total premature birth. The positive rate of the extremely preterm birth related with chorioamnionitis was $72 \%$ and the positive rates in clinic and histological chorioamnionitis were $5.5 \%$ and $66.5 \%$, which were significantly higher than those in early preterm birth, moderate preterm birth and late preterm birth. Histologic chorioamnionitis cases have no clinical symptoms. The positive rates of increased white blood cells count, neutrophils count and increased level of C-reactive protein in histological chorioamnionitis are $37.6 \%, 39.4 \%, 64.2 \%$ and 0 $\%$ respectively, which are significantly lower than those in clinic chorioamnionitis $(88.9 \%, 100 \%, 100$ $\%, 11.1 \%$, respectively, $\mathbf{p}<\mathbf{0 . 0 5}$ ). The top three pathogens are ureaplasma urealytium, escherichia coli and group B streptococcus. $90.9 \%$ of the extremely preterm born have severe neonatal complications; 63.6\% have septicemia and shared chorioamnionitis with the mother. Chorioamnionitis is an important risk factor of extremely preterm birth. The positive rate of the extremely preterm birth with chorioamnionitis is significantly higher than that early, modulate and late preterm birth. Histologic chorioamnionitis is the main type of chorioamnionitis, which may induce poor prognosis of the extremely preterm birth newbornsy.
\end{abstract}

Key words: Extremely preterm birth, chorioamnionitis, clinic research

Preterm birth is one of the leading causes of neonatal death. Based on reports of previous studies, the incidence of preterm birth is over $5 \%$ worldwide and every year about 15 million preterm birth may occur globally ${ }^{[1-3]}$. In recent years, the incidence of preterm birth has shown an increasing tendency. It was estimated that the preterm birth rate is about 5 to $10 \%$ in Europe and the US, where the preterm birth rate is $10 \%{ }^{[4,5]}$. Unfortunately, the pathogenesis of preterm birth is still unclear; therefore, to further explore the underlying mechanism is of great importance for the prevention and treatment of the preterm birth.

For the preterm infants, the risks of mortality and morbidity are strongly associated the degree of prematurity. Extremely preterm birth refers to the premature birth before $28 \mathrm{w}$, although it accounting for only a small percentage of all deliveries, extremely preterm can lead to significant increase in motility and morbidity rate during the perinatal period ${ }^{[6,7]}$. In developed countries, extremely preterm birth is the major contributor to neonatal deaths, while in China, the data regarding extremely preterm birth have not been regularly collected and there is few systematic clinical research.

Chorioamnionitis (CAM) a commonly observed infection during the period of pregnancy and previous studies, including a recent study by our research group suggested that CAM is a risk factor of preterm birth ${ }^{[8-10]}$. Therefore, in the present study, we analyzed the clinical data of 164 cases of extremely preterm birth and 
discussed the relationship between extremely preterm birth and chorioamnionitis (CAM) and their effect on both mother and fetus, providing the assistance for the clinical treatment of extremely preterm birth and improving prognosis of both mother and fetus.

\section{MATERIALS AND METHODS}

\section{Patients:}

A total of 164 cases of extremely preterm birth who have hospitalized in Fujian Provincial Maternity and Children's Hospital from January $1^{\text {st }} 2017$ to June $31^{\text {st }}$ 2018 were enrolled in this study. The mean maternal age of the women with extremely preterm birth is $31.0 \pm 5.1 \mathrm{y}$ old (range from 18.0 to 45.0 ) and the mean gestational age of $(23.0 \pm 2.2) \mathrm{w}(20.0$ to 27.6$) \mathrm{w}$. The 164 cases of the patients including 129 cases of singleton pregnancy, 32 cases of gemellary pregnancy and 3 cases of triplet pregnancy, a total of 202 extremely preterm babies were delivered, with the mean weight at birth of (640.2 \pm 262.6$) \mathrm{g}$ (235 to 1340) g, including 104 cases of male babies and 98 cases of female babies.

\section{Diagnostic criteria of premature delivery and ex- tremely preterm birth:}

The diagnosis criteria of premature delivery were as follow: Women who deliver babies at the pregnancy period of less than $37 \mathrm{w}$. The lower limit of premature delivery varied among different countries and regions, the premature delivery of $20 \mathrm{w}$ is used as the lower limit in the United States ${ }^{[11]}$; and the premature delivery of $22 \mathrm{w}$ is used as the lower limit in the Europe ${ }^{[12]}$. The lower limit of premature delivery gestational weeks in China still has used the definition made by World Health Organization (WHO) in 1960s-the cases with the pregnancy period of $28 \mathrm{w}$ or the weight of the babies at birth $\geq 1000 \mathrm{~g}$; Along with the improvement in the obstetrics and treatment level of newborn, increasing number of preterm babies have been positively treated and the research on extremely preterm birth treatment has been gradually valued, therefore the delivery from $20 \mathrm{w}$ to $36^{+6} \mathrm{w}$ is used as the diagnosis boundary in the present study. According to the delivery gestational weeks, the preterm infants were divided into: extremely preterm birth $(<28 \mathrm{w})$, very preterm birth $\left(28\right.$ to $\left.31^{+6} \mathrm{w}\right)$, moderate preterm birth $\left(32\right.$ to $\left.33^{+6} \mathrm{w}\right)$, late preterm birth (34 to $36^{+6} \mathrm{w}$ ).

\section{Diagnostic criteria of CAM:}

The diagnostic criteria of CAM were followed as described previously ${ }^{[13,14]}$ : Placental pathology examination can detect the increase of maternal neutrophil granulocyte in fetus surface of placenta and amniotic membrane andCAM canbedivided into clinical chorioamnionitis and histological chorioamnionitis (h-CAM). The diagnostic criteria of clinical CAM were: temperature rise of pregnant woman $\left(\geq 37.8^{\circ}\right)$, pulse increase ( $\geq 100$ times/min), fetal heart rate increase ( $\geq 160$ times/min), fundus tenderness, unpleasant odor of vaginal secretion, rise in peripheral blood leucocyte count ( $\left.\geq 15 \times 10^{9} / \mathrm{L}\right)$ and more than 2 symptoms or signs accompanied with the temperature rise of pregnant woman can be detected. No above symptoms but only placental pathology manifestations are shown in h-CAM. Newborn's severe complications ${ }^{[15]}$ : mainly including cerebroventricular heaemorrhage at Stages III and IV, periventricular leukomalacia, necrotizing enterocolitis, bronchopulmonary dysplasia, septicemia, premature infant retinopathy etc.

\section{Study variables:}

The clinical symptoms of the participators, including body temperature, heart rate, uterine pain, fetal heart rate was recorded and after delivery, the neonatal survival rate, birth weight, number of days in intensive care unit treatment, severe complications were also recorded.

\section{Laboratory tests:}

The blood routine test, serum level of C-reactive protein CRP, cervical pathogen culture and procalcitonin were analyzed by the clinical laboratory of Fujian Provincial Maternity and Children's Hospital.

\section{Statistical analysis:}

The data were analyzed by SPSS 19.0 statistical software. The quantitative data were presented as mean $\pm \mathrm{SD}$ and the enumeration data is described by the absolute number, rate and composition ratio. The difference between the groups was tested by chi-square test, and the $* \mathrm{p}<0.05, * * \mathrm{p}<0.01$ were considered as statistically significant.

\section{RESULTS AND DISCUSSION}

From January $1^{\text {st }} 2017$ to June $31^{\text {st }} 2017$, there were a total of 22490 cases of women making delivery in our hospital ( $\geq 20 \mathrm{w}$ ) (21751 cases of single pregnancy, 728 cases of gemellary pregnancy, 11 cases of triple pregnancy) and 23240 cases of delivered new- 
borns. There were 2281 premature births (1878 cases of singleton pregnancy, 393 cases of twin pregnancy, 10 cases of triple pregnancy), 2694 cases of premature delivery, the premature birth rate of $10.1 \%$ (fig. 1A, 2281/22490) and premature newborns accounted for $11.6 \%$ of newborns (fig. 1B, 2694/23240). Among the premature cases, there were 164 cases of extremely preterm birth, 322 cases of very preterm birth, 379 cases of moderate preterm birth, and 1416 cases of late preterm birth. The incidence of extremely preterm birth was $0.7 \%(164 / 22490)$, accounting for $7.2 \%$ of preterm birth (164/2281), the data were shown in fig. 1C.

Among the 146 cases of extremely preterm birth, there were 118 cases with CAM and the positive rate of CAM was $72.0 \%$ (118/164), including 9 cases of clinical CAM and 109 cases of h-CAM. The clinical CAM positive rate of extremely preterm birth was $5.5 \%(9 / 164)$,

A

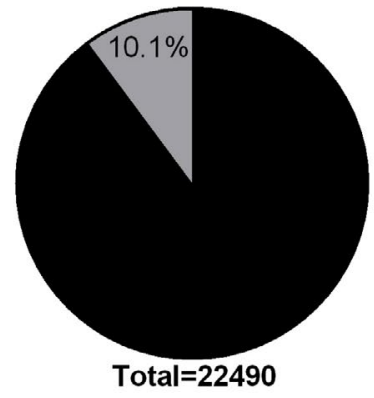

B

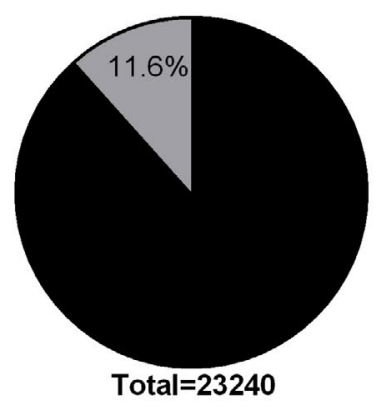

C

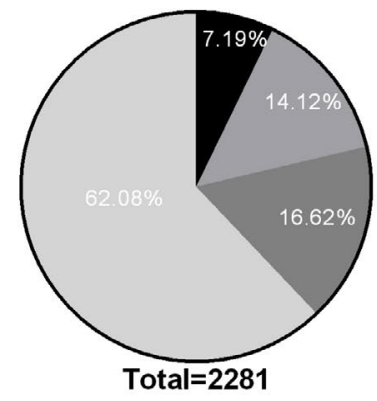

Fig. 1: The incidence rate of extremely preterm birth A. The premature birth rate among 22490 women making delivery; $B$. The percentage of premature newborns among 23240 newborns; $C$. The percentage of extremely preterm birth, very preterm birth, moderate preterm birth and late preterm birth among the newborns.

Special Issue 3, 2021 which was significantly higher than the clinical CAM positive rate of very preterm birth, moderate preterm birth and late preterm birth $(4.0 \%, 4.5 \%$ and $1.0 \%$ respectively), the difference was statistically significant $(\mathrm{p}<0.01)$; h-CAM positive rate of extremely preterm birth was $66.5 \%(109 / 164)$, which was also higher than the h-CAM positive rate of very preterm birth, moderate preterm birth and late preterm birth $(41.9 \%$, $33.0 \%$ and $18.5 \%$ respectively) and the difference is statistically significant (Table $1, \mathrm{p}<0.01$ ).

Among the 9 women with extreme preterm birth and clinical CAM, all of them had fever, and 3 of them (33\%) has shown increased fetus heart rate, 1 of them $(11.1 \%)$ had uterine pain, odd-smell vaginal discharge and septicopyemia. Moreover, the percentage of women with increased white blood cells (WBC) count, increased neutrophils (NE) count, increased C-reactive protein (CRP) and increased procalcitonin were $88.9 \%, 100.0 \%, 100.0 \%$, and $11.1 \%$, respectively. On the other hand, the 109 women with extreme preterm birth and h-CAM showed on clinical symptoms of infection and the percentage of women with increased WBC count, increased NE count, increased CRP and increased procalcitonin were $37.6 \%, 39.4 \%$, $64.2 \%, 0.0 \%$, respectively. The above index of extreme preterm birth with h-CAM was significantly lower than women with clinical CAM (Table 2, $\mathrm{p}<0.05$ ). The cervical pathogen positive rate of women with CAM and h-CAM cervical were $55.6 \%$ and $38.8 \%$, respectively. Ureaplasma urealyticum, Escherichia coli and B group streptococci were the top three pathogens found in the cervical pathogen culture product.

The extremely preterm babies accounted for $0.9 \%$ of newborns (202/23250), $7.5 \%$ of premature babies $(202 / 2694)$, due to financial burden and long-term sequelae of extreme preterm babies, 180 cases in which the families gave up rescue and 11 cases survived in neonatal treatment of 22 cases with the survival rate of $50 \%(11 / 22)$. Survivors had a minimum weight of $730 \mathrm{~g}$ at birth and a minimum gestational age of $26^{+3}$ w. $90 \%(10 / 11)$ of the survivors had severe neonatal complications, $63.6 \%(7 / 11)$ of which had neonatal sepsis, $72.7 \%(8 / 11)$ of which had brain damage and $63.6 \%(7 / 11)$ of which had premature retinopathy, requiring surgery, $63.6 \%(7 / 11)$ of which had bronchial dysplasia, $36.4 \%(4 / 11)$ of which had necrotizing enterocolitis and $9.1 \%(1 / 11)$ of which had ventricular hemorrhage at Stage III. All of the survived extremely preterm babies with maternal CAM had neonatal 
TABLE 1: THE POSITIVE RATE OF CAM AMONG WOMEN WITH PRETERM BIRTH

\begin{tabular}{lcccc}
\hline & \multicolumn{2}{c}{ Clinical CAM } & \multicolumn{2}{c}{ h-CAM } \\
\cline { 2 - 5 } & Positive & Negative & Positive & Negative \\
\hline Extremely preterm birth & $9(5.5 \%)$ & $155(94.5 \%)$ & $109(66.5 \%)$ & $55(33.5 \%)$ \\
Very preterm birth & $13(4.0 \%)$ & $309(96.0 \%)$ & $135(41.9 \%)$ & $187(58.1 \%)$ \\
Moderate preterm birth & $17(4.5 \%)$ & $362(95.5 \%)$ & $125(33.0 \%)$ & $254(67.0 \%)$ \\
Late preterm birth & $14(1.0 \%)$ & $1402(99.0 \%)$ & $262(18.5 \%)$ & $1154(81.5 \%)$ \\
$x^{2}$ value & \multicolumn{2}{c}{30.324} & \multicolumn{2}{c}{220.848} \\
p value & \multicolumn{2}{c}{0.000} & \multicolumn{2}{c}{0.000} \\
\hline
\end{tabular}

TABLE 2: COMPARISON OF THE CLINICAL SYMPTOMS AND RESULTS OF LABORATORY TESTS EXTREME PRETERM BIRTH WOMEN WITH CLINICAL CAM AND H-CAM

\begin{tabular}{lccccc}
\hline & \multicolumn{2}{c}{ Clinical CAM $(\mathbf{n}=9)$} & \multicolumn{2}{c}{ h-CAM $(\mathbf{n}=109)$} & \multirow{2}{*}{$\mathrm{p}$ value } \\
\cline { 2 - 5 } & $\mathrm{n}$ & Percentage $(\%)$ & $\mathrm{n}$ & Percentage $(\%)$ & \\
\hline Fever & 9 & $100.0(9 / 9)$ & 0 & $0(0 / 109)$ & $<0.0001$ \\
Increased fetal heart rate & 3 & $33.3(3 / 9)$ & 0 & $0(0 / 109)$ & $<0.0001$ \\
Uterine pain & 1 & $11.1(1 / 9)$ & 0 & $0(0 / 109)$ & 0.0005 \\
Odd-smell vaginal discharge & 1 & $11.1(1 / 9)$ & 0 & $0(0 / 109)$ & 0.0005 \\
Septicopyemia & 1 & $11.1(1 / 9)$ & 0 & $0(0 / 109)$ & 0.0005 \\
Increased WBC count & 8 & $88.9(8 / 9)$ & 41 & $37.6(41 / 109)$ & 0.0027 \\
Increased NE count & 9 & $100.0(9 / 9)$ & 43 & $39.4(43 / 109)$ & 0.0004 \\
Increased CRP & 9 & $100(9 / 9)$ & 54 & $64.2(54 / 84)$ & 0.0294 \\
Increased procalcitonin & 1 & $11.1(1 / 9)$ & 0 & $0(0 / 50)$ & 0.0174 \\
Cervical pathogen positive & 5 & $55.6(5 / 9)$ & 33 & $38.8(33 / 85)$ & 0.3307 \\
\hline
\end{tabular}

sepsis and CAM-free extreme preterm babies had no intrauterine infections such as sepsis. Survived extremely preterm babies had normal binaural hearing test, the average number of days in the neonatal intensive care unit was $41 \mathrm{~d}$.

In the present study, we analysis the incidence of extremely preterm birth based on Chinese population and also explore the relationship between extremely preterm birth and chorioamnionitis. We found that chorioamnionitis is a risk factor of extremely preterm birth and histologic chorioamnionitis, which is the main type of chorioamnionitis among the extremely preterm birth, may lead to poor prognosis of the newborns.

Premature birth is one of the most common and severe complications of pregnancy and results of the previous studies suggested that extremely preterm birth can lead to high motility and morbidity of the newborns ${ }^{[9,12]}$. The incidence rate of extremely preterm birth varies between different countries and Blencowe et al. ${ }^{[16]}$ analyzed the premature birth data in 184 countries, indicating that premature babies accounted for $11 \%$ of all live births and the extremely preterm newborns account for $5 \%$ of preterm babies. However, studies on the extremely preterm birth in China are very limited and the incidence rate of extremely preterm birth in China has not yet been reported. In the present study, we found that the extreme preterm birth rate was $0.7 \%$ among 22490 women and extreme preterm newborns accounted for $7.5 \%$ of premature newborns. These results suggested that the incidence of extremely preterm birth in China is slightly higher than other countries. This may because in China, obstetricians and gynaecologists have insufficient awareness or insufficient attention to the severity and prevention of extremely preterm birth and the clinical therapies for the disease has yet to be clarified and standardized, leading to increased incidence rate of extremely preterm birth.

Intrauterine infection accounts for about $25 \%$ to $40 \%$ of all cases of premature birth ${ }^{[17]}$ and it was hypothesized that pathogens travels from the lower genital tract or through the placenta blood line or by other means to the uterus, invading the decidua, chorioamnion, amniotic fluid and even involving the fetus, causing placental CAM, fetal inflammatory syndrome, etc. The release of the inflammatory factors can induce uterine contraction, premature rupture of membranes and cervical ripening leading to premature birth. Some previous studies suggested that the positive rate of preterm h-CAM before 30 to $32 \mathrm{w}$ was about $33 \%$ to $83 \%^{[18]}$. In the present study, we observed that the positive rate of CAM in extreme preterm birth is $72.0 \%$, which was significantly higher than that at other stages of preterm birth and interestingly, we found that among the 
women with preterm birth, the premature gestational age is negatively associated with the positive rate of CAM, indicating that CAM is a risk factor of extremely preterm birth.

CAM can be divided into two types, including clinical CAM and h-CAM. Clinical CAM was first defined by Lenki et al. ${ }^{[14]}$. According to clinical manifestations and laboratory tests, it can be diagnosed before delivery. Results of previous study showed that clinical CAM is already the final stage of CAM, which may lead to poor prognosis of mother and the newborns ${ }^{[18]}$. In the present study, we found that 9 of extreme preterm birth women with CAM were clinical CAM and they have shown the clinical symptoms of fever, increased fetal heart rate and other clinical manifestations; results of the laboratory tests have shown increased WBC count, NE count and increased CRP; in one sever case, the patient has the symptoms of septicopyemia, infectivity shock and other severe complications. Thus, our data suggested that to avoid undesired clinical outcomes, in the clinical field, obstetricians should make rapid clinical decisions for the treatment of extreme preterm birth women with clinical CAM.

In the present study, we found that CAM-related extreme preterm birth was mainly characterized by h-CAM, which accounted for $66.5 \%$ of the extreme preterm birth. Unlike clinical CAM, h-CAM has no typical infection manifestations such as fever, uterine pain and increased fetal heart rate. Thus, the diagnosis of h-CAM is difficult. A previous study proposed the idea of monitoring the serum biomarkers, for example maternal peripheral blood CRP for the early diagnosis of h-CAM during pregnancy ${ }^{[10]}$. In our study, we found that the positive rate of peripheral blood CRP, white blood cell count and other laboratory indicators in h-CAM maternal blood was lower than that of clinical CAM. These results suggested that h-CAM may not be simply diagnosed by the routine blood test and physicians must be alert to infection factors when treating the pregnant women of $<28 \mathrm{w}$ with preterm birth symptoms, perfect infection-related examinations and carefully identify and interpret relevant indicators. Besides the routine blood test, the examination of cytokines, for example interleukin- $6^{[13]}$, interleukin- $8^{[18]}$, tumor necrosis factor alpha ${ }^{[13]}$ in cervical or amniotic fluid may be helpful for the early diagnosis of h-CAM and thus decrease the risk of preterm birth.

Stout et al. ${ }^{[19]}$ found that the proportion of pregnant women with placental bacterial infection accounting for pregnant women with extremely advanced preterm birth was significantly higher than that that of the pregnant women who make term delivery. Common bacteria include Escherichia coli, streptococcus and anaerobic bacteria. Ohlin et al. ${ }^{[20]}$ found that extreme preterm babies are at risk of neonatal sepsis and staphylococci are the most common pathogens. Combs et al. ${ }^{[21]}$ detected 305 cases of spontaneous preterm labor and intact fetal membranes of amniotic fluid 16s ribosomal DNA and amniotic fluid culture, finding that the most common pathogen is Ureaplasma urealyticum. In this research, the positive rates of clinical CAM and h-CAM-related extreme preterm birth were $55.6 \%$ and $38.8 \%$, respectively. The first three pathogens were Ureaplasma urealyticum, Escherichia coli and Group B streptococci. These results were consistent with previous observation. However, the traditional methods for the pathogen culture is limited by the collection of specimens, culture media and other factors, therefore the detection rate is not high, the relationship between viruses, fungi and extreme preterm birth has not been clear yet. Along with the development of molecular biology, the application of PCR technology and gene sequencing technology, the detection rate of pathogens will be improved to further deepen the understanding of CAM-related extreme preterm birth pathogens.

Currently, more than $90 \%$ of the extreme preterm babies can survive in developed countries, but in developing countries, the survival rate of extreme preterm babies may significantly decreased, and for some very low-income countries, it can reach to less than $10 \%{ }^{[12]}$. Researches have shown that the survived extremely preterm babies often have severe neonatal complications $^{[15]}$. In the present study, for most of the extreme preterm babies, especially those under the age of $26 \mathrm{w}$, their families gave up the rescue, there for the survival rate of the rescued extreme preterm babies was $50 \%$, the minimum gestational age of which was $26^{+3} \mathrm{~W}$ and the incidence of severe complications such as neonatal sepsis, brain damage, premature babies retinopathy and bronchial dysplasia was very high. The results showed that the mortality rate and severe complication incidence of extreme preterm babies in Fujian, China were markedly higher than those in development countries, which may be mainly due to the negative response of the family for the treatment and the unsatisfied ability of the physicians for the treatment of extreme preterm.

We found that the incidence rate of sepsis among the survived extremely preterm babies in this research was as high as $63.6 \%$ and in all of the cases, maternal CAM 
was diagnosed. Interestingly, cases without CAM had no infectious complications, such as sepsis. Previous reports suggested that the survived extremely preterm babies with maternal CAM often had fetus inflammatory reaction symptoms and sepsis, which may cause shortterm and long-term sequela and increase the risks of bronchopulmonary dysplasia, necrotizing enterocolitis, Periventricular Leukomalaci, cerebral palsy ${ }^{[18]}$. Ohlin et $a .^{[20]}$ found that $66 \%$ of extreme preterm babies had sepsis, which was associated with severe bronchial dysplasia. The previous finding and our results indicateed that CAM can cause extreme preterm birth and also can aggravate the poor prognosis of extreme preterm babies.

To sum up, CAM is an important risk factor of extremely preterm birth and CAM among extremely preterm birth women is mainly h-CAM, which may lead to poor prognosis of extremely preterm babies. To our knowledge, this is the first report on the relationship between extremely preterm birth and CAM that based on Chinese population. Although our results need to be further investigated with larger sample size, our data provide novel evidence for the diagnosis and treatment of extremely preterm birth in China.

\section{Acknowledgements:}

This study was supported by the Sponsored by Fujian provincial health technology project (2019-ZQN-24).

\section{Conflicts of interest:}

The authors report no conflicts of interest.

\section{REFERENCES}

1. Barker C, Dunn S, Moore GP, Reszel J, Lemyre B, Daboval T. Shared decision making during antenatal counselling for anticipated extremely preterm birth. Paediatr Child Health 2019;24:240-9.

2. Barbone AS, Li X, Arabin B, Kira Y, Jani JC, Cannie MM. Preliminary modeling of effective positioning of Arabin cerclage pessary in women at high risk of preterm birth. Ultrasound Obstet Gynecol 2020;55:557-8.

3. Torgalkar R, Dave S, Shah J, Ostad N, Kotsopoulos K, Unger $\mathrm{S}$, et al. Multi-component lipid emulsion vs. soy-based lipid emulsion for very low birth weight preterm neonates: a prepost comparative study. J Perinatol 2019;39:1118-24.

4. Griffin JB, Jobe AH, Rouse D, McClure EM, Goldenberg RL, Kamath-Rayne BD. Evaluating WHO-recommended interventions for preterm birth: a mathematical model of the potential reduction of preterm mortality in sub-Saharan Africa. Glob Health Sci Pract 2019;7:215-27.

5. Kovac U, Jasper EA, Smith CJ, Baer RJ, Bedell B, Donovan
$\mathrm{BM}$, et al. The association of polymorphisms in circadian clock and lipid metabolism genes with 2nd trimester lipid levels and preterm birth. Front Genet 2019;10:540.

6. Simmons LE, Rubens CE, Darmstadt GL, Gravett MG. Preventing preterm birth and neonatal mortality: exploring the epidemiology, causes, and interventions. Semin Perinatol 2010;34:408-15.

7. Goldenberg RL, Culhane JF, Iams JD, Romero R. Epidemiology and causes of preterm birth. Lancet 2008;371:75-84.

8. Qiu L, Pan M, Zhang R, Ren K. Maternal peripheral blood platelet-to-white blood cell ratio and platelet count as potential diagnostic markers of histological chorioamnionitis-related spontaneous preterm birth. Journal of clinical laboratory analysis 2019;33:e22840.

9. Palmsten K, Nelson KK, Laurent LC, Park S, Chambers CD, Parast MM. Subclinical and clinical chorioamnionitis, fetal vasculitis and risk for preterm birth: a cohort study. Placenta 2018;67:54-60.

10. Stojanovska V, Miller SL, Hooper SB, Polglase GR. The Consequences of preterm birth and chorioamnionitis on brainstem respiratory centers: implications for neurochemical 2018;12:26.

11. American College of Obstetricians and Gynecologists. Practice bulletin No. 171: management of preterm labor. Obstet Gynecol 2016;128:e155-64.

12. Di Renzo GC, Cabero Roura L, Facchinetti F, Helmer $\mathrm{H}$, Hubinont C, Jacobsson B, et al. Preterm labor and birth management: recommendations from the European Association of Perinatal Medicine. J Matern Fetal Neonatal Med 2017;30:2011-30.

13. Kayem G, Batteux F, Girard N, Schmitz T, Willaime M, Maillard F, et al. Predictive value of vaginal IL-6 and TNF $\alpha$ bedside tests repeated until delivery for the prediction of maternal-fetal infection in cases of premature rupture of membranes. Eur J Obstet Gynecol Reprod Biol 2017;211:8-14.

14. Lencki SG, Maciulla MB, Eglinton GS. Maternal and umbilical cord serum interleukin levels in preterm labor with clinical chorioamnionitis. Am J Obstet Gynecol 1994;170:1345-51.

15. Anderson JG, Baer RJ, Partridge JC, Kuppermann M, Franck LS, Rand L, et al. Survival and major morbidity of extremely preterm infants: a population-based study. Pediatrics 2016;138.

16. Blencowe H, Cousens S, Oestergaard MZ, Chou D, Moller AB, Narwal R, et al. National, regional, and worldwide estimates of preterm birth rates in the y 2010 with time trends since 1990 for selected countries: a systematic analysis and implications. Lancet 2012;379:2162-72.

17. Vornhagen J, Quach P, Boldenow E, Merillat S, Whidbey C, Ngo LY, et al. Bacterial hyaluronidase promotes ascending GBS infection and preterm birth. MBio 2016;7(3).

18. Yoneda S, Shiozaki A, Ito M, Yoneda N, Inada K, Yonezawa $\mathrm{R}$, et al. Accurate prediction of the stage of histological chorioamnionitis before delivery by amniotic fluid IL-8 level. Am J Reprod Immunol 2015;73:568-76.

19. Stout MJ, Conlon B, Landeau M, Lee I, Bower C, Zhao Q, et al. Identification of intracellular bacteria in the basal plate of the human placenta in term and preterm gestations. Am J 
Obstet Gynecol 2013;208:e226-7.

20. Ohlin A, Björkman L, Serenius F, Schollin J, Källén K. Sepsis as a risk factor for neonatal morbidity in extremely preterm infants. Acta Paediatr 2015;104:1070-6.

21. Combs CA, Gravett M, Garite TJ, Hickok DE, Lapidus J, Porreco R, et al. Amniotic fluid infection, inflammation, and colonization in preterm labor with intact membranes. J Obstet Gynecol 2014;210:125-e1.
This is an open access article distributed under the terms of the Creative Commons Attribution-NonCommercial-ShareAlike 3.0 License, which allows others to remix, tweak, and build upon the work non-commercially, as long as the author is credited and the new creations are licensed under the identical terms

This article was originally published in a special issue, "Evolutionary Strategies in Biomedical Research and

Pharmaceutical Sciences" Indian J Pharm Sci 2020:83(3)

Spl issue;42-48 